\title{
Wide FoV and large pupil 'active' Schmidt telescope toward a space based UHE neutrino Observatory and a highly sensitive Observatory of the Earth
}

\author{
P. Mazzinghi \\ Istituto Nazionale di Ottica \\ Largo Enrico Fermi, 6 - 50125 Firenze (Italy) \\ E-mail: piero.mazzinghieino.it \\ P. Sandri \\ OHB-Italia SpA \\ via Arno 108, 50019 Osmannoro, Sesto Fiorentino (FI) - Italy \\ E-mail: psandri@cgspace.it
}

\author{
P. Spillantini ${ }^{1}$ \\ Agenzia Spaziale Italiana \\ Via del Politecnico snc, 00133, Roma, Italy \\ E-mail: spillantiniefi.infn.it
}

\begin{abstract}
The possible realization of the wide FoV and large pupil Schmidt recently proposed for a space instrument opens the possibility of realizing even more large pupil optical telescopes in space. This development is important for the realization of space observatories dedicated to the observation of the terrestrial surface and events in the terrestrial atmosphere, as well for astronomical observations requiring a high sensitivity and good resolution on a very wide FoV. Optical systems of $50^{\circ} \mathrm{FoV}$ with pupils of tens of meters diameter can be obtained with a huge primary mirror of a Schmidt telescope by assembling and opening in space several 'active' sub-mirrors of relatively large dimensions. For fundamental research in physics it is of paramount importance the detection and measurement of the fluorescence light emitted by particle showers produced by extreme energy primary cosmic rays, and among them detection and measurement of events produced by ultra-high energy neutrinos, in particular in the energy range of $10^{18}-10^{19} \mathrm{eV}$ (the so called cosmogenic neutrinos produced by the energy loss of charged cosmic rays produced at large distance $(>100$ $\mathrm{Mpc}$ ) from the Earth). Some examples of such possible optical systems are evaluated and discussed in detail.
\end{abstract}

35th International Cosmic Ray Conference - ICRC2017-

10-20 July, 2017

Bexco, Busan, Korea

\footnotetext{
${ }^{1}$ Speaker

(c) Copyright owned by the author(s) under the terms of the Creative Commons

Attribution-NonCommercial-NoDerivatives 4.0 International License (CC BY-NC-ND 4.0).
} 


\section{Introduction}

In last years significant progresses have been achieved in the development of technology of lightweight and deployable large mirror for space telescopes. For optical systems of 'astronomical class' these issues have been solved for JWST (Ref. [1]) using 18 beryllium rigid mirror segments, each one $1.32 \mathrm{~m}$ in diameter for a total diameter of $6.6 \mathrm{~m}$. The segments are deployed and aligned in orbit with the help of six actuators for each rigid segment. The large cost of manufacturing large beryllium mirrors limits the extension of this technology to larger diameters. In last decade ESA supported two projects for using normal glass, a thin sheet of ZERODUR®ceramic glass, for achieving a better surface quality and deployability with the same areal weight. The first ALC (Advanced LIDAR Concept) (Ref. [2], [3], [4] and [5]) studied the general concept of a deployable, lightweight, $4 \mathrm{~m}$ aperture, space borne telescope for LIDAR application, producing also a technology demonstrator. The following project LATT (Large Aperture Telescope Technology) (Ref. [6] and [7]) extended the study, producing an Optical demonstrator Bread Board (OBB), made by a $400 \mathrm{~mm}$ diameter, $1 \mathrm{~mm}$ thick, ZERODUR ${ }^{\circledR}$ glass, coupled to a backplane by 19 actuators controlling the optical surface. The long stroke of the actuators, about $1 \mathrm{~mm}$, guarantees the optical performances, compensating mechanical deployment errors and other deformation due to the thermal and mechanical environment. This technology makes then possible the construction of virtually unlimited mirrors, using the same mechanisms presently used for the deployment of large microwave antennas.

\section{Design and performance of a $50^{\circ} \mathrm{FoV}$ and $10 \mathrm{~m}$ pupil 'active' Schmidt telescope for space}

Based on this concept it was presented at the "Cosmic Ray Origin: Beyond the standard model' conference in S. Vito di Cadore in September of last year, the proposal of an 'EUSOlike' experiment as precursor of a ultra-high energy neutrino space observatory (Ref.[8]). In December the EUSO collaboration decided to adopt a Schmidt optical system (Ref. [9]) for the optics of the proposed K-EUSO experiment. Detail of the optical system were discussed in the following months between the Optics Working Group (OWG) and the Russian component of the EUSO collaboration producing the project presented to this conference (Ref. [10]).

Based on these premises, there are all the elements for expanding the K-EUSO optical system to a larger diameter by extending the pupil area up to $10 \mathrm{~m}$ as an intermediate step toward a possible optics for a neutrino space observatory.

The layout of the telescope, based on the 'Double Donut Schmidt Camera' (DDSC) layout for the K-EUSO UHECR detector ((Ref. [10]), is reported in Fig. 1. Its FoV is $50^{\circ}$ and it is composed by the primary mirror $18 \mathrm{~m}$ diameter, segmented in several elements (Fig. 2) deployable to fit the fairing launcher and a thin and light deployable correcting disc of $10 \mathrm{~m}$ in diameter. In fig. 1 are traced also the rays. The nominal values of the tilt and decentering of the mirror can be met in orbit by the use of the LATT technology, strongly mitigating a potential criticality. In fact, even in a simplified version (three actuators per segment) LATT allows for the recovering of the tilt and decentering of each mirror segment due to thermal variation or micro-vibrations. The first order parameters of the telescope are summarized in Table 1. The polychromatic RMS spot radius and the corresponding ground resolution are reported in fig.3. 
Wide FoV and large pupil 'active' Schmidt telescope toward a space based UHE neutrino Observatory ...

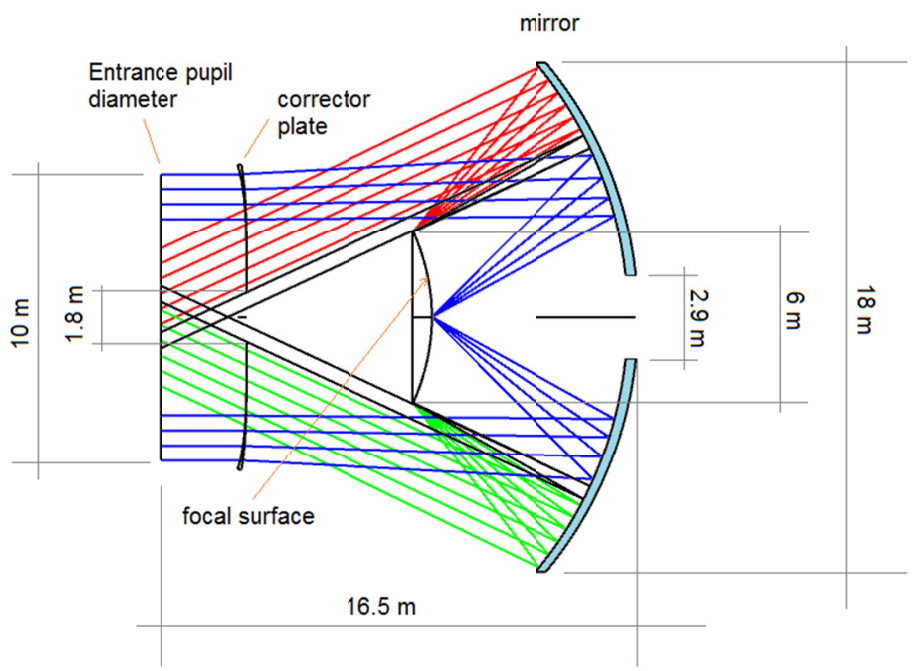

Fig. 1. Layout of a large mirror telescope based on the DDSC layout.
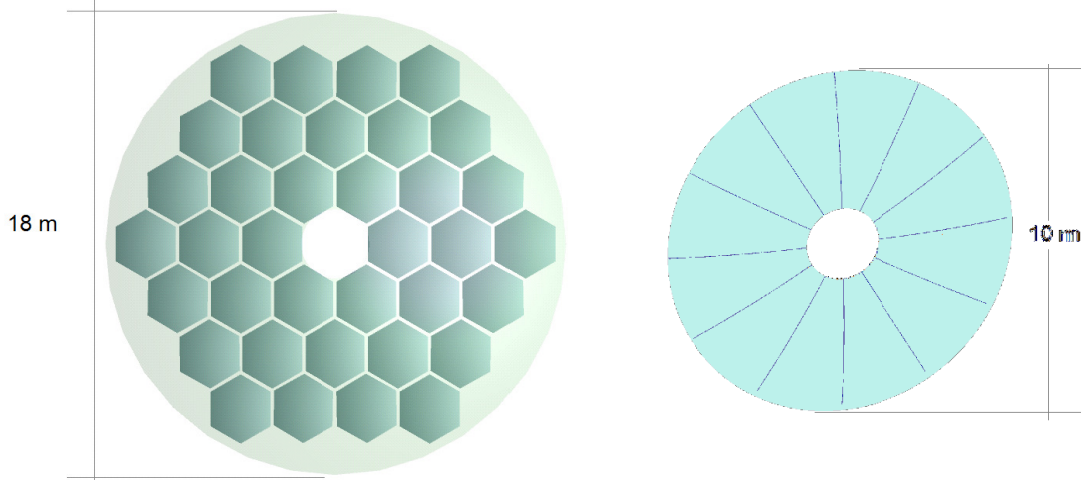

Fig. 2. Left: frontal view of the segmented mirror. Right view of the segmented corrector plate (Ref. [9]).

\begin{tabular}{|l|l|}
\hline Parameter & Value \\
\hline FoV & $50^{\circ}$ \\
\hline Entrance pupil diameter & $10 \mathrm{~m}$ \\
\hline Effective focal length $f$ & $7 \mathrm{~m}$ \\
\hline Relative aperture & $\mathrm{F} / 0.7$ \\
\hline Ground resolution (GSD) from $400 \mathrm{~km}$ & $0.5 \mathrm{~km}<\mathrm{GSD}<1.0 \mathrm{~km}$ \\
\hline
\end{tabular}

Table 1. First order optical parameters.

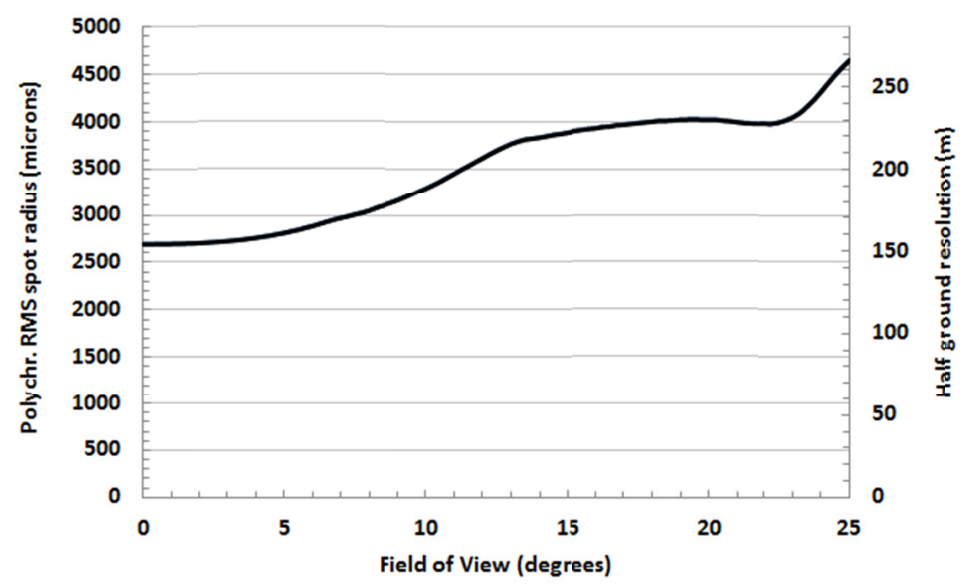

Fig. 3. Polychromatic RMS spot radius and corresponding half ground resolution from altitude of $400 \mathrm{~km}$. 
With the DDSC layout reported in Fig. 1 it is possible to obtain a high rate of UHECR expected events as indicated in Table 2 for different altitudes of the orbit. The number of possible cosmogenic neutrino events (dependent from the distribution assumed for their source in the universe (Ref. [11], [12] and [13]) can supply information for dimensioning a future dedicated neutrino observatory. Besides making possible to realize a powerful observatory of UHECR beyond the GKZ limit it can be considered an intermediary step needed for optimizing the parameters of the final neutrino observatory.

\begin{tabular}{|c|c|c|c|}
\hline Altitude $(\mathrm{km})$ (circular orbit) & 400 & 800 & 1200 \\
\hline Total FoV $\left(^{\circ}\right)$ & 50 & 50 & 50 \\
\hline Radius of spherical surface $(\mathrm{km})$ & 198 & 378 & 580 \\
\hline Area of spherical surface $\left(10^{3} \mathrm{~km}^{2}\right)$ & 110 & 450 & 1066 \\
\hline Mass of atmosphere $\left(10^{3} \mathrm{t}\right)$ & 1100 & 4500 & 10660 \\
\hline Pupil diameter $(\mathrm{m})$ & 10 & 10 & 10 \\
\hline E threshold (EeV) & 1.2 & 4 & 10 \\
\hline Proton events/year (uniform source distribution $+\mathrm{GKZ}$ ) with $\mathrm{E}_{\mathrm{p}}>100 \mathrm{EeV}$ & 70 & 290 & 680 \\
\hline Neutrino events/year $($ Kalashek $\approx$ min) $($ ref.11) & 0.3 & 1.3 & 4.5 \\
\hline Neutrino events/year $($ Kalashek $\approx$ Max) $($ ref.11) & 4 & 16 & 36 \\
\hline Neutrino event/year (Ahlers bestfit) (ref.12) & 0.04 & 0.2 & 0.4 \\
\hline Neutrino events/year (A+H p=100\%) (ref.13) & 0.002 & 0.01 & 0.02 \\
\hline Neutrino events/year $(\mathrm{A}+\mathrm{H}$ p=10\%) (ref.13) & -- & 0.001 & 0.002 \\
\hline
\end{tabular}

Table 2. UHECR and neutrino expected events with $\mathrm{FoV}=50^{\circ}$ and pupil $=10 \mathrm{~m}$.

\section{Applications to an highly sensitive Earth observation}

Furthermore the optical system of very wide FoV and a large pupil above discussed constitutes an interesting step forward in the application to other problematics requiring a good angular resolution $(<0.1$ degrees) on a very wide FoV.

\subsection{A lidar for Earth observation and diagnostic}

The first to be mentioned is the earth observation and diagnostic with lidar systems, item that was at the origin of the above mentioned LATT development, and will benefit from a large area telescope. According to the generalized lidar equation (Ref. [14])

$$
P(r)=E_{0} \frac{c A}{2 r^{2}}\left[\frac{3}{8 \pi} \beta_{m}(r)+\frac{p(\pi, r)}{4 \pi} \beta_{a}(r)\right] e^{-2 \int_{0}^{r} \beta_{e}\left(r^{\prime}\right) d r^{\prime}}+M(r)+b
$$

the power $P(r)$ on the detector is proportional to the laser energy $E_{0}$ and the collecting area $A$, and inversely proportional to the square of target distance. The distance of an earth observing system, even in LEO, is at least $400 \mathrm{~km}$, much more than that of ground based lidars. Moreover, being the laser power on a spacecraft limited by many factors, like electrical power, weight and dimensions of the laser itself, current generation of spaceborne lidars are, in fact, limited to the simplest application, the backscattering rangefinder, where the backscattering cross section $\beta$ is quite high. Even if this kind of system can give high precision data, as the ground distance and altitude of the cloud top, advanced lidars, like Differential Absorption Lidar (DIAL) and Fluorescence Lidar, could deliver much more information, useful both for the proposed mission as well as, in general, for global earth monitoring.

The use of large mirror telescope was already proposed for the above mentioned applications: a DIAL operating at $935 \mathrm{~nm}$ could deliver the columnar content of $\mathrm{CO}_{2}$ along the atmosphere (Ref. [3], [4] and [15]). A Lidar devoted to the retrieval of the Chlorophyll 
fluorescence spectrum in the 650-750 nm range (Ref. [16] and [17]) could deliver information on the photosynthetic rate and then on the net biomass production and $\mathrm{CO}_{2}$ uptake from vegetation.

Both applications are in the top list for future missions by ESA and other agencies: in the selection of ESA's eighth Earth Explorer mission, the two candidate opportunity missions were CarbonSat, aiming to the determination of the global distribution of carbon dioxide and methane the atmosphere, and FLEX (Fluorescence Explorer, presently selected), aiming to provide global maps of vegetation fluorescence to retrieve the actual photosynthetic activity.

The proposed neutrino observatory could be easily integrated with one (or even both) these applications, simply installing a suitable laser on the back of the focal plane and transmitted through the hole of the Schmidt corrector, using the zero field detector (or a suitable dedicated one in that position) for signal retrieval. If the main detector could be used also for those applications, the laser could be scanned through the whole FoV obtaining an imaging lidar. The integration of those two (or three) mission will considerably reduce the overall development, launch and operation costs, increasing the operation duty cycle and, at the end, their feasibility.

\subsection{Pushing to a sub-arcmin angular resolution}

As a general observation we must remark that the potentialities of the Schmidt camera are surprising. Despite its very simple layout, consisting of few elements, it can collect a large as 50 degrees field of view working with an entrance pupil of $10 \mathrm{~m}$ or more. The focal surface is oriented in opposite direction with respect to the observed scene, thus providing an intrinsic protection from the stray light, both in-field and out-of-field. The limited number of elements (optical and mechanical) contributes in minimizing the stray-light level to level as $10^{-4} \div 10^{-5}$ (see Ref.[9] and [10]).

Moreover for those applications where a higher resolution is needed, the Schmidt camera can be optimized to provide sub-arcmin angular resolution. Fig. 4 shows the DDSC optimized to a reduced $\mathrm{FoV}$ of 24 degrees presenting an angular resolution beyond the arcmin in the spectral range extending from $337 \mathrm{~nm}$ to $391 \mathrm{~nm}$ (see Fig. 5). It is clear a classic concept of optics: the smaller the observed FoV the higher the angular resolution.

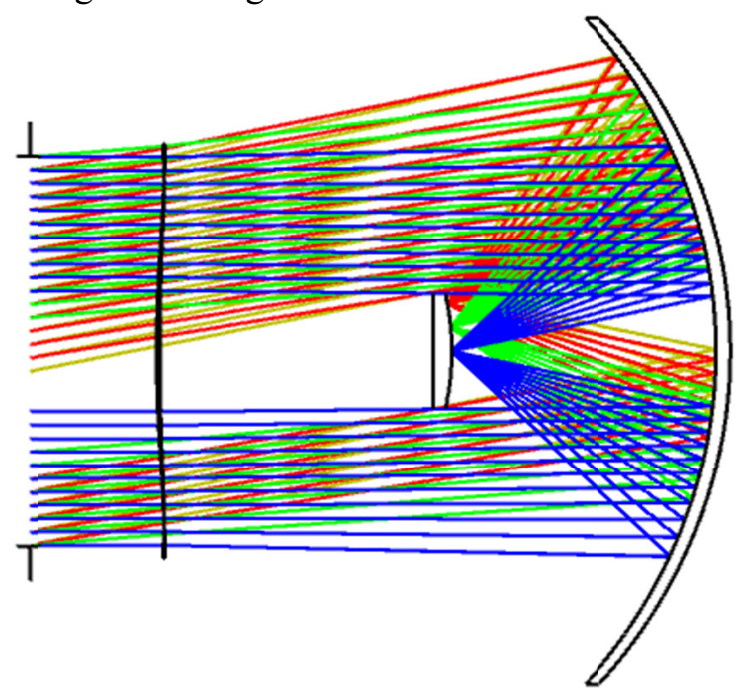

Fig. 4. DDSC optimized for a FoV of 24 degrees. 


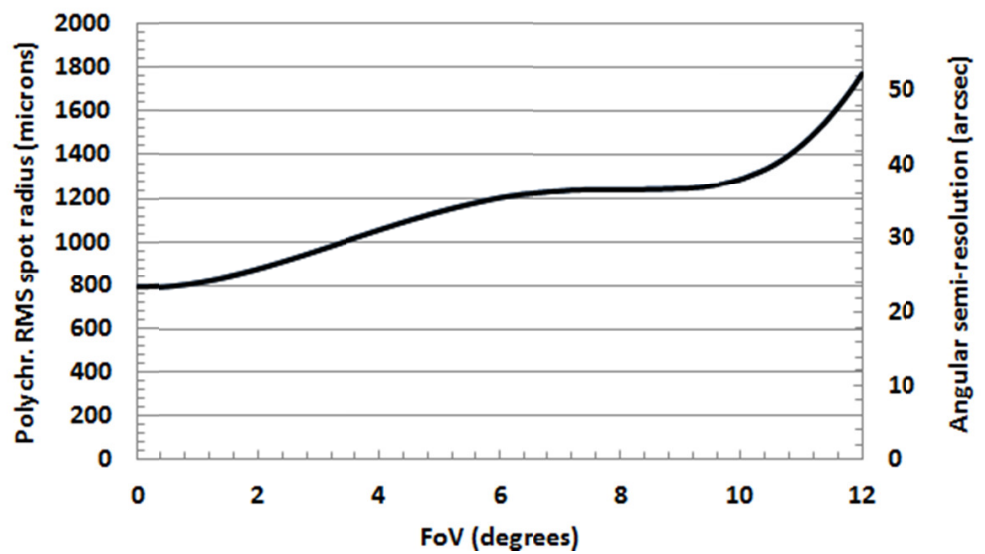

Fig. 5. RMS spot radius and angular semi-resolution of the DDSC optimized for a FoV of 24 degrees.

Last but not least the Schmidt camera can be manufactured, integrated, launched into space and maintained aligned in the on-orbit environment. Thanks to the segmented concept adopted both for the mirror and for corrector plate, (see Fig. 2), the Schmidt camera has no limits from a manufacturing and integration point of view. Besides the impact of the manufacturing and integration tolerances on the performances has been analyzed (see. Ref.[9]). The most critical tolerances in terms of performances are the tilt and decentering of each mirror segment. This criticality is fully solved by the adoption at mirror level of the LATT technology (Ref.[6] and [7]), which, also in a very simplified (i.e. low consumption) form, allows for the recovering of a nominal alignment even in presence of thermal gradients.

With an adequate angular resolution and a very large pupil a wide FoV system allows to complement the debris localization and tracking devices operating from ground: a device equipped with such an optical system, flying in the debris environment will observe them in situ and better profit of the sun light reflection.

\section{Conclusions}

The proposed technology will allow a step forward in the detection of extreme energy cosmic rays, and neutrinos, including the so called cosmogenic neutrinos. Even if the proposed telescope is much larger than any existing orbiting telescope, the combined effect of the moderate optical resolution required by the experiment and the availability of new technology for the main mirror allows its construction with reasonable cost and high probability of success. The telescope, derived from the classical Schmidt camera concept, has been specifically designed for extreme low weight and deployability, compatible with the existing class of launchers, like Ariane $\mathrm{V}$. The tolerance analysis shows that the design performances can be maintained after deployment in space. The active technology used for the main mirror will guarantee the optical performances also with the variable thermal load in low/medium Earth orbit.

A further reduction in the mission cost could be achieved exploiting the synergy with other missions currently under development. Especially lidar missions will benefit of the large collecting area, allowing the use of lower power lasers, or even make possible missions not achievable elsewhere. A typical example is FLEX, the mission for the measurement of the vegetation fluorescence recently selected by ESA for Earth Explorer. FLEX uses a passive technology, the atmospheric oxygen absorption bands, for the detection of the fluorescence excited by the sun, but this implies several limitations in the detection wavelength and in the time of day for the measurement. The direct laser excitation, installing a relatively moderate power laser in the pro- 
posed telescope, will remove such limitations and the mission could cooperate with FLEX, acting as calibration tool.

\section{References}

[1]. J.P. Gardner, J.C. Mather, M. Clampin et al. Space Sci Rev (2006) 123: 485.

[2]. P. Mazzinghi, V. Bratina, D. Ferruzzi, L. Gambicorti, F. Simonetti, A. Zuccaro Marchi, P. Salinari, F. Lisi M. Olivier, A. Bursi and J. Pereira do Carmo "An Ultra-Lightweight, Large Aperture, Deployable Telescope for Advanced Lidar Applications", A. Wilson, ed., Proc. 6th Internat. Conf. on Space Optics, ESTEC, Noordwijk, The Netherlands, ISBN 93-9092-932-4, ISSN 1609-042X, ESA SP-621 (2006).

[3]. F. Simonetti, A. Zuccaro Marchi, L. Gambicorti, P. Mazzinghi and V. Bratina, "Large aperture telescope for advanced lidar system”, Optical Engineering, Vol. 49, Issue 7, pp. 073001 073001-10 (2010).

[4]. P. Mazzinghi, V. Bratina, D. Ferruzzi, L. Gambicorti, F. Simonetti, A. Zuccaro Marchi, P. Salinari, F. Lisi, M. Olivier, A. Bursi, D. Gallieni, R. Biasi and J. Pereira, "Deployable, lightweight and large aperture space borne telescope for LIDAR based earth observations", Proceedings of SPIE, Vol. 6750, pp. 0Y-1 0Y10, (2007).

[5]. P. Mazzinghi, V. Bratina, D. Ferruzzi, L. Gambicorti, F. Simonetti, A. Zuccaro Marchi, P. Salinari, F. Lisi, M. Olivier, A. Bursi, D.Gallieni, R. Biasi and J. Pereira, "Lightweight active controlled primary mirror technology demonstrator", Proceedings of SPIE, Vol. 6750, pp. 0X-1 0X10, (2007).

[6]. R. Briguglio, R. Biasi, D. Gallieni, C. Vettore, F. d'Amato, et al., "Laboratory demonstration of a primary active mirror for space with the LATT: large aperture telescope technology", Proc. SPIE 9904, Space Telescopes and Instrumentation (2016).

[7]. R. Briguglio, C. Arcidiacono, M. Xompero, F. Lisi, A. Riccardi, et al. "The LATT way towards large active primaries for space telescopes", Proc. SPIE 9904, Space Telescopes and Instrumentation (2016).

[8]. P. Spillantini, P. Mazzinghi and P. Sandri "A EUSO-like experiment as a precursor of a ultrahigh energy neutrino Space Observatory", submitted to Nuclear and Particle Physics Proceedings 00 (2017) 1-6.

[9]. P. Sandri, P. Mazzinghi and V. Da Deppo "The Double Donut Schmidt Camera, a wide field, large aperture and light weight space telescope for the detection of ultra-high energy cosmic rays", in preparation. (presented to the Tokyo collaboration meeting, Dec. 2016).

[10]. P. Sandri, S.Sharakin, P. Mazzinghi and Y. Takizawa, "Schmidt type optical system for the KLYPVE-EUSO UHECR detector", presented to this conference.

[11]. O.E. Kalashek, V.A. Kuzmin, D.V. Semokov and G. Sigl, "Ultra-High Energy Neutrino Fluxes and Their Constraints", Physical Review D 66 (2002) 063004.

[12]. M. Ahlers et al. 'GZK neutrinos after Fermi-LAT diffuse photon flux measurement', Astropart. Phys. 34 (2010) 106.

[13]. M. Ahlers and F. Halsen 'Minimal Cosmogenic Neutrinos', arXiv:1208.4181v1, 21 Aug 2012.

[14]. P. Piironen and E. W. Eloranta, "Demonstration of a high-spectral-resolution lidar based on an iodine absorption filter," Opt. Lett. 19, 234-236 (1994).

[15]. P. Mazzinghi, V. Bratina, D. Ferruzzi, L. Gambicorti, F. Simonetti, A. Zuccaro Marchi, P. Salinari, F. Lisi M. Olivier, A. Bursi, J. Pereira do Carmo" An Ultra-Lightweight, Large Aperture, Deployable Telescope for Advanced Lidar Applications" A. Wilson, ed., Proc. '6th Internat. Conf. on Space Optics', ESTEC, Noordwijk, The Netherlands, ISBN 93-9092-932-4, ISSN 1609-042X, ESA SP-621 (2006). 
Wide FoV and large pupil 'active' Schmidt telescope toward a space based UHE neutrino Observatory ...

[16]. P. Mazzinghi, G. Cecchi, R. Guzzi: "Feasibility of Active Remote Sensing from Space" 2nd International Workshop on Remote Sensing of Vegetation Fluorescence, Canadian Space Agency, Montréal, Canada November 17,19 (2004).

[17]. R. Guzzi, P. Mazzinghi, G. Cecchi, V. Bratina, A. Zuccaro Marchi, D. Ferruzzi, F. Simonetti, L. Gambicorti, M. Olivier, A. Bursi, F. Lisi, P. Salinari, D. Gallieni, J. Pereira Do Carmo "Advanced LIDAR technologies for active remore sensing of vegetation fluorescence from space", Proceedings 3rd International Workshop on Remote Sensing of Vegetation Fluorescence, Florence, Italy (2007). 